\title{
Diagnosis of Bearing Damage in Mechanical Equipment Combining Fuzzy Logic Variable Phase Layered Algorithm
}

\author{
Yao CHEN
}

\begin{abstract}
The paper aims at the problem that the bearing of mechanical equipment affects the safe, stable and efficient operation of mechanical equipment. In this paper, a fuzzy logic variable phase layered algorithm (flvpla) is proposed. The dimension reduction is realized by calculating the vibration signal. The vibration signal is effectively used to diagnose bearing fault, and the signal value is reduced to conduction fault classification. Finally, the experimental results show that the dimension reduction effect based on flvpla is better than that based on principal component analysis (PCA) algorithm and LTSA. The fault recognition rate of ba-svm is significantly higher than that of genetic algorithm optimized support vector machine (GA-SVM) and particle swarm optimization support vector machine (PSO-SVM). Therefore, the combination of flvpla and ba-svm can obtain higher recognition accuracy.
\end{abstract}

Keywords: fault diagnosis; fuzzy logic variable phase layered algorithm (flvpla); multiscale permutation entropy; rolling bearing

\section{INTRODUCTION}

With the constant progress of the social economy, the demand for the quantity and quality of equipment in the industry, as an important sector supporting the national economy, has also continuously increased [1-3]. As an essential part of many mechanical devices and equipment, bearings directly affect the safe, stable, and efficient operation of mechanical equipment. Hence, controlling the operation of bearing components with precision is one of the crucial guarantees for the normal operation of machinery. At present, there are relatively many methods for diagnosing bearing faults. For example, based on the analysis of vibration signals, the vibration signals often show nonlinearity, so nonlinear analysis methods are of higher practical significance than linear analysis methods in extracting fault information due to the complexity of the mechanical system [3-8]. In recent years, many nonlinear analysis methods have been used to the feature extraction of bearing vibration signals, such as approximate entropy, sample entropy, permutation entropy, and multiscale entropy [9-10]. Some scholars have put forward a method based on empirical mode decomposition and neural network, which is characterized by focusing on the diagnosis of fault types afterward. In addition, some scholars established a bearing fault diagnosis method based on the LSSVM migration learning method, which has effectively improved the prediction accuracy of the model. However, it still depends on the quantity and quality of the input data to a large extent [11-12]. Other scholars classify the bearing life into several stages in the bearing life status identification method based on probabilistic principal component analysis and carry out prediction accordingly. However, such methods cannot determine the status of adjacent stages reasonably and accurately, which can easily lead to incorrect determination [13-15]. In these determination methods, the extraction of features is mainly used to determine the health of the bearing. In order to ensure the comprehensiveness of the information, it is generally necessary to extract the multi-dimensional fault feature set. However, it may lead to the "curse of dimensionality in classification" to the subsequent pattern identification at the same time [16-17].
Hence, an original high-dimensional feature set is established through the calculation of the eigenvalue of the vibration signal based on the fuzzy logic variable phase layered algorithm in this paper. The fault classification is carried out on the low-dimensional feature set after the dimensionality reduction so as to determine whether there are any faults in the bearing and implement the classification of bearing fault models more properly.

\section{FUZZY LOGIC VARIABLE PHASE LAYERED ALGORITHM (FLVPLA)}

Fuzzification refers to the conversion of a precise value of the input variable into a linguistic variable value (fuzzy input value) in the appropriate domain. Given the actual traffic situation, for the convenience of design and calculation, the membership function of the input and output fuzzy subsets adopts the curve based on the triangle correction. There are seven values for curve and language variables, that is, ES, VS, S, M, L, VL, and EL.

In the system proposed in this paper, the "IF A AND B THEN C" type of reasoning method is adopted. For example, the $i$-th rule is described as the following:

IF $l_{i}$ is $L$ and $l_{i+1}$ is $L^{\prime}$ THEN $e$ is $e$

The Cartesian product of this rule is shown as the following:

$$
R_{i}=l_{i} \times l_{i+1} \times e_{i}
$$

As there are a total of $7 \times 7=49$ rules, the total fuzzy relationship matrix can be written in the following form:

$$
R=\underset{i=1}{\vee} R_{i}
$$

It is assumed that the vehicle queue length of the current phase main queue at an intersection at time $t$ is $l_{1}$ $(t)$, and the subsequent phase main queue vehicle queue length is $l_{2}(\mathrm{t})$. After quantization and fuzzification processing, the obtained language variable values are $L_{1}(t)$ and $L_{2}(t)$. Subsequently, the output of the fuzzy controller at this moment can be obtained according to the fuzzy synthesis rule (in general, the maximum-minimum rule) as 
the following:

$$
E(t)=\left(L_{1}(t) \times L_{2}(t)\right) \cdot R
$$

After the fuzzy output obtained by this equation is calculated and processed by the weighting method, a fuzzy look-up table will be finally formed from the green delay time of the current phase obtained. In practical control, the response speed can be improved simply by looking up the table.

The essential concept of the Ensemble Empirical Mode Decomposition (hereinafter referred to as EEMD for short) is to add the Gaussian white noise to the raw signals and then carry out the EMD processing a number of times. The purpose of adding the white noise to the raw signals is to make use of the uniform frequency distribution of white noise to eliminate the intermittent phenomenon in the raw signals so as to prevent the mode mixing issue after signal decomposition. After the raw signals added with the white noise are processed by EMD, the decomposed IMF components will contain random noise signals. Since the white noise can cancel each other out after multiple averages, the mean value for the IMF components acquired after EMD processing many times is taken to eliminate the effect of white noise. It is assumed that there is a signal matrix $\boldsymbol{X}(m \times n)$. After principal component analysis of the signal matrix, the following can be obtained:

$$
\boldsymbol{X}=t_{1} P_{1}^{T}+t_{2} P_{2}^{T}+\cdots+t_{j} P_{j}^{T}+\cdots+t_{r} P_{r}^{T}+E
$$

In the above equation: $t_{j}$ stands for a vector of $m \times 1$, which indicates the $j$-th principal element; $P_{j}$ stands for a vector of $n \times 1$, which indicates the $j$-th load vector; and $\boldsymbol{E}$ stands for the residual matrix. Let the principal element matrix be $\boldsymbol{T}(m \times r)$ and the load matrix be $\boldsymbol{P}(n \times r)$, then $\boldsymbol{X}$ can be expressed as the following

$$
\boldsymbol{X}=\boldsymbol{T} \boldsymbol{P}^{T}+\boldsymbol{E}
$$

Thus, its projection on the residual subspace can be obtained as the following:

$$
\overline{\boldsymbol{X}}=\boldsymbol{X}\left(I-P P^{T}\right)
$$

In order to monitor the abnormal condition of the data, the principal component analysis process mainly detects whether an abnormality has occurred during the operation of the equipment by calculating two indicators, that is, the statistic $T^{2}$ and the squared prediction error $S P E$.

$T^{2}$ can be used to detect the changes of sample data in the principal component subspace and characterize the degree of deviation of the fault information from the normal model during the state monitoring process. The statistics of $T^{2}$ are defined as the pivot score, and the calculation process is shown as the following:

$$
\left\{\begin{array}{l}
\boldsymbol{T}=\boldsymbol{X} \boldsymbol{P}_{m} \\
\overline{\boldsymbol{X}}=t \boldsymbol{P}_{m}^{T}=\boldsymbol{X} \boldsymbol{P}_{m} \boldsymbol{P}_{m}^{T} \\
e=\boldsymbol{X}-\overline{\boldsymbol{X}}=\boldsymbol{X}\left(I-\boldsymbol{P}_{m} \boldsymbol{P}_{m}^{T}\right)
\end{array}\right.
$$

The principal component score value $\boldsymbol{T}$ of the sample matrix can be calculated based on the above equation to measure the estimated value $\overline{\boldsymbol{X}}$ and the residual $e$. Thus, the principal component statistical value can be obtained as the following:

$T^{2}=t^{T} \Lambda^{-1} t$

In the above equation: $\boldsymbol{\Lambda}$ stands for a diagonal matrix composed of the first $m$ eigenvalues of the covariance matrix, $\boldsymbol{\Lambda}=\operatorname{diag}\left(\lambda_{1}, \lambda_{2}, \ldots, \lambda_{m}\right)$, in which $m$ stands for the number of principal elements.

The threshold value $T_{\text {lim }}^{2}$ of $T^{2}$ follows the $F$ distribution $F(m, n-m)$ with degrees of freedom $m$ and $n-m$. Let the number of sample sampling points be $n$, and the significance experiment level be $a$, then the following can be obtained:

$T_{\text {lim }}^{2}=\frac{m\left(n^{2}-1\right)}{n-m} F_{a}(m, n-m)$

The squared prediction error SPE can be calculated according to the equation as the following:

$S P E=\|\bar{X}\|^{2}$

Its threshold value can be expressed as the following:

$$
\begin{aligned}
& S P E_{\lim }=\theta_{1}\left[\frac{c_{a} h_{0} \sqrt{2 \theta_{2}}}{\theta_{1}}+1+\frac{\theta_{2} h_{0}\left(h_{0}-1\right)}{\theta_{1}^{2}}\right]^{1 / h} \\
& h_{0}=1-2 \theta_{1} \theta_{3} /\left(3 \theta_{2}^{2}\right) \\
& \theta_{i}=\sum_{j=r+1}^{n} \lambda_{j}^{i}
\end{aligned}
$$

In the above equation: $c_{a}$ stands for the statistical value of the standard normal distribution of the confidence level a; $\lambda_{\mathrm{j}}$ stands for the $j$-th eigenvalue of the covariance matrix of $\boldsymbol{X}$.

In the coarse-grained process of multiscale permutation entropy, only the coarse-grained model that starts to transform with the first data is taken into consideration based on the time scale factor, whereas the coarse-grained mode that starts transforming with other data in the time scale is neglected. As a result, the entropy value fluctuates significantly with the increase of the scale factor, and the direct value error increases with the rise in the scale factor.

In view of the limitations of the coarse-grained transformation in the calculation of multiscale permutation and the influence of shorter time series on permutation entropy calculation, CMPE can be used to address the issue. The main ideas are as the following: Firstly, the permutation of different coarse-grained sequences is calculated under the same scale factor, and then the mean value of all permutation entropies under this scale factor is obtained and used as the composite multiscale permutation 
entropy under this scale factor. The specific steps are described as the following.

1) For the time series $\{x(i), i=1,2, \ldots, N\}$, the following equation is used to establish a coarse-grained sequence:

$y_{k}^{\tau}=\left\{y_{k, 1}^{\tau}, y_{k, 2}^{\tau}, \cdots, y_{k, p}^{\tau}\right\} ; p=[N / \tau]$

$y_{k, j}^{\tau}=\frac{1}{\tau} \sum_{i=(j-1) \tau+k}^{j_{\tau}+k-1} x_{i}, 1 \leq i \leq p, 1 \leq k \leq \tau$

2) The permutation entropy EPE of the coarse-grained sequence is calculated under any scale factor $\tau$, and then the mean value of the $\tau$ permutation entropies is obtained and used as the composite multiscale permutation entropy $E_{C M P E}$ under the $\tau$ scale, that is, the following can be obtained:

$E_{C M P E}(x, \tau, m)=\frac{1}{\tau} \sum_{k=1}^{\tau} E_{P E}\left(y_{k}^{\tau}, m\right)$

The mean permutation entropy of the coarse-grained sequence calculated under each scale factor is used to replace the multiscale permutation entropy to characterize the complexity and randomness of the time series under various scale factors.

\subsection{Local Tangent Space Alignment Algorithm}

The steps of the local tangent space alignment algorithm are described as the following:

1) Select the neighborhood. The neighborhood of each sample point $x_{i}$ is calculated and denoted as $x_{i}=\left[x_{i 1}, x_{i 2}, \ldots\right.$, $\left.x_{i k}\right]$, which indicates $\mathrm{k}$ nearest neighbor points including $\mathrm{x}_{\mathrm{i}}$.

2) Local linear projection. The neighborhood $X_{i}$ corresponding to any sample point $x_{i}$ is used to establish a matrix $X_{i}-\bar{x}_{i} I_{k}^{T}$, and the vector corresponding to the largest $d$ singular values of the matrix is reconstituted into a matrix $\boldsymbol{V}_{i}$.

3) Permutation of local coordinate system. The matrix $\Phi=\sum_{i=1}^{N} S_{i} W_{i} W_{i}^{T} S_{i}^{T} \quad$ in the permutation matrix is calculated. Among them, $S_{i}$ stands for the selection matrix, $S_{i}=\left\{x_{i 1}, \ldots, x_{i k}\right\} ; \quad \boldsymbol{W}_{i}=\boldsymbol{I}-\left[\boldsymbol{I}_{k} / \sqrt{k}, \boldsymbol{V}_{i}\right]\left[\boldsymbol{I}_{k} / \sqrt{k} \boldsymbol{V}_{i}\right] ;$ $S_{i} \in R^{N \times k}, \boldsymbol{I}_{\boldsymbol{k}}$ stands for the local transformation matrix; $\boldsymbol{I}$ stands for the identity matrix. The eigenvectors $u_{1}, \ldots, u_{d}$ correspond to the d smallest non-zero eigenvalues in the calculation, then $T=\left[u_{1}, \ldots, u_{d}\right]^{\mathrm{T}}$ is the calculated embedding result.

\subsection{Improved Semi-Supervised Local Tangent Space Alignment Algorithm}

In the dimensionality reduction process, the category label information of the samples has not been used to improve the identification accuracy of the compressed feature set. In practical mechanical engineering applications, it is often easier to obtain the fault category label information of some samples. In general, the manifold structures of similar samples are usually the same, while the manifold structures of heterogeneous samples are different. Hence, the rational use of sample category label information can better reflect the high-dimensional data manifold structure. Hence, semi-supervised learning is combined with the local tangent space alignment algorithm.

In the process of constructing the neighborhood diagram, the Euclidean distance measurement method between sample points is used in the local tangent space alignment algorithm to select the neighborhood points, which can only represent the positional relationship between the sample points. In addition, it is possible that the samples of the same type and heterogeneous samples are selected as the neighbor points. Hence, a new, improved distance measurement method that integrates the concept of semi-supervised learning is put forward, which has combined cosine similarity with Euclidean = distance, that is, the following can be obtained:

$d_{i j}=\left(\frac{1-d_{i j}^{c}}{2}\right) \times d_{i j}^{m} ; i, j=1,2, \cdots, n$

In the above equation: $d_{i j}^{c}$ and $d_{i j}^{m}$ stand for the cosine similarity of $x_{i}$ and $x_{j}$ and the Euclidean distance, $d_{i j}^{c} \in[-1,1],\left(1-d_{i j}^{c}\right) / 2 \in[0,1]$.

It is assumed that there is a high-dimensional fault data feature set $X=\left\{X_{1}, X_{2}\right\}$, in which $X_{1}=\left[\left(x_{1}, l_{1}\right),\left(x_{2}, l_{2}\right), \ldots\right.$, $\left.\left(x_{m}, l_{c}\right)\right], \mathrm{m}$ stands for the number of samples containing category value information,$l_{i}(i=1,2, \ldots, c)$ stands for the category of sample point $x_{i}, c$ stands for the number of categories; $X_{2}=\left[x_{m+1}, x_{m+2}, \ldots, x_{m+n}\right]$, and $n$ stands for the number of samples without category information. In the process of constructing the neighborhood diagram, each sample contains more samples of the same kind, the more accurate the local space is obtained. Hence, the compressed feature set after dimensionality reduction can reflect the high-dimensional spatial manifold structure of the sample data more appropriately. Thus, the distance matrix based on semi-supervised learning can be defined as the following:

$$
D=\left\{\begin{array}{l}
\sqrt{1-e^{-d_{i j}^{2} / \bar{d}}} ; l_{i}=l_{j} \\
\sqrt{1+e^{d_{i j}^{2} / \bar{d}}} ; l_{i} \neq l_{j} \\
\left(\sqrt{1-e^{-d_{i j}^{2} / \bar{d}}}+\sqrt{1+e^{d_{i j}^{2} / \bar{d}}}\right) / 2 ; \\
x_{i} \text { and } x_{j} \text { without label }
\end{array}\right.
$$

In the above equation: $d_{i j}$ stands for the improved distance between $x_{i}$ and $x_{j} ; D$ stands for the improved distance matrix reconstructed based on semi-supervised learning; and $\bar{d}$ stands for the mean value of $d_{i j}$.

For the samples with the same category label, the distance is compressed to the interval $[0,1]$; for the samples with different labels, the distance is stretched to the interval $[\sqrt{2},+\infty]$; for the samples with the special 
category label, the distance is between the aforementioned two. Hence, in the process of constructing the neighborhood graph, this distance metric can be used to increase the probability that samples with the same category label are selected as neighbors.

Support vector machine is a special machine learning method for studying limited sample prediction. It has the features of strong generalization capacity and optimal global convergence. However, it often requires that the penalty parameter $C$ and the Gaussian kernel function $\sigma$ should be set manually, which can affect its classification performance. Hence, the bat algorithm with better global optimization performance is used to optimize the support machine parameters.

The bat algorithm (hereinafter referred to as BA for short) is a search algorithm that simulates bats in using the sonar system for hunting down prey and avoiding obstacles. The specific process of the application of the BA algorithm to optimize support vector machine parameters is shown in Fig. 1 as the following. The specific steps are described below:

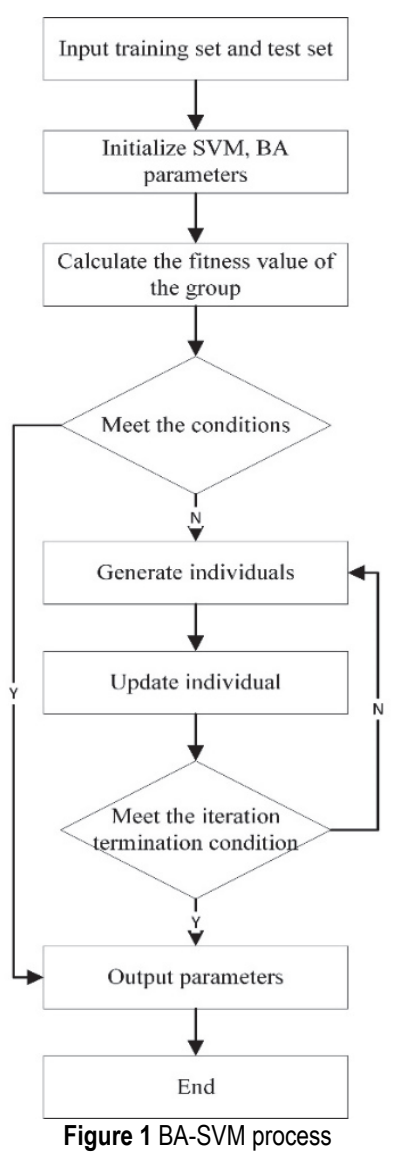

1) Initialize the position of bats, number of individuals, pulse frequency range, loudness, and other parameters, and set the value range of SVM parameters $(C, \sigma)$.

2) Calculate the fitness value of each bat. Take each bat in the bat colony as the optimized parameter combination $(C, \sigma)$ of the support vector machine, and use the training samples to establish the prediction model. Subsequently, the trained model is used for the identification of experiment samples, and the correct identification rate of each bat is returned as the corresponding fitness value.

3) Generate new bat individuals. Update the pulse frequency based on $F_{i}=F_{\min }+\left(F_{\max }-F_{\min }\right) \times R$ and, in which $F_{i}$ stands for the frequency of the sound wave $\left(F_{\min }\right.$ stands for the minimum value, and $F_{\max }$ stands for the maximum value); $R$ and $[0,1]$ stands for a randomly generated number. Subsequently, the flight speed $v(i)$ of bats is updated according to $v_{i}^{t+1}=v_{i}^{t}+\left(x_{i}^{t}-x_{i}^{t+1}\right)=x_{i}^{t}+v_{i}^{t+1}$, and new bat individuals are finally generated based on $h x_{i}^{t+1}=x_{i}^{t}+v_{i}^{t+1}$.

4) Update bat individuals. If $R$ and $1>R(i)$ is met, then a new individual can be obtained according to $R^{t+1}(i)=$ $R^{0}(i) \times[1-\exp (-\gamma t)]$ random perturbation of the optimal bat individual $x_{\text {best }}$ in the current population. Subsequently, the current bat is replaced with the new bat individual. If $R$ and $2<A(i)$ and $f\left(x_{\text {new }}(i)\right)>f\left(x_{\text {old }}(i)\right)$ are met, the old bat at the current position is replaced with the new bat individual, and the pulse loudness $R$ is updated according to $A^{t+1}(i)=$ $\alpha \times A^{t}(i)$ and $R^{t+1}(i)=R^{0}(i) \times[1-\exp (-\gamma t)]$. In addition, the pulse rate is $A(i)$. Among them, $\alpha$ and $\gamma$ are constants and generally the value 0.9 is taken.

5) The algorithm terminates when the maximum number of iterations is reached, and the optimal SVM parameters are output. Otherwise, proceed to step 3 to continue the operation.

\subsection{Diagnosis of Bearing Fault}

On the basis of the theory described above, the CMPE, FLVPLA, and BA-SVM are combined for the diagnosis of bearing faults. The diagnosis process is shown in Fig. 2 below. The specific steps are described as the following:

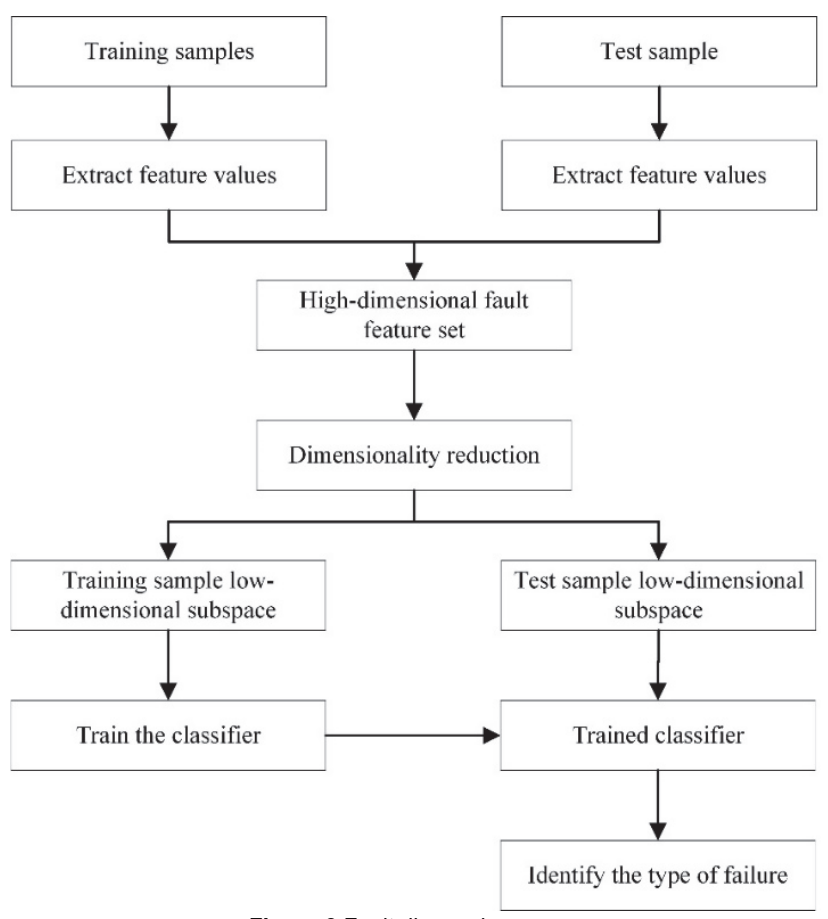

Figure 2 Fault diagnosis process

1) Collect the vibration signals of the rolling bearing in different working states, collect $p$ samples in each working state, randomly select a as the training sample, and the remaining $p$ - $a$ samples are used as the experiment sample.

2) Calculate the CMPE of the original data to obtain $q$ 
eigenvalues for each sample, and establish the eigenvector matrix $\boldsymbol{R} \boldsymbol{p} \times \boldsymbol{q}$.

3) In order to avoid the issues of time-consuming training and information redundancy due to the excessively high dimension of the fault feature set, the FLVPLA method is used to reduce the dimensionality of the highdimensional fault feature set of the training sample and the experiment samples and obtain the $d$ dimensional space manifold.

4) Use the d-dimensional feature subset of the training samples for BA-SVM training, and then apply the trained BA-SVM model to carry out pattern identification on the experiment samples and determine the type of faults.

\section{EXPERIMENT VERIFICATION}

In order to verify the effectiveness and practicability of the method proposed in this paper, different experimental data are selected for analysis.
The experimental bearing is a 1210 self-aligning ball bearing with an inner diameter of $50 \mathrm{~mm}$, an outer diameter of $90 \mathrm{~mm}$, and a width of $20 \mathrm{~mm}$. SG bimetallic electric engraving machine is used to simulate pitting corrosion faults on the surface of steel ball, the surface of the inner and outer ring channels. The fault diameter is about $5 \mathrm{~mm}$, and the depth is about $0.2 \mathrm{~mm}$.

The BVT- 5 bearing vibration measuring instrument is used to acquire the vibration signal of the experiment bearing through the acceleration sensor. The radial and axial load of the bearing are 300 and $100 \mathrm{~N}$, respectively. The spindle speed is $1800 \mathrm{r} / \mathrm{min}$, the sampling frequency is $5120 \mathrm{~Hz}$, and the number of sampling points is 2048 . Fifty groups each of 4 kinds of working state data are detected, with a total of 200 groups of vibration signal samples, and the time-domain waveforms of the vibration signals of the bearing in the four types of working states are shown in Fig. 3 as the following.

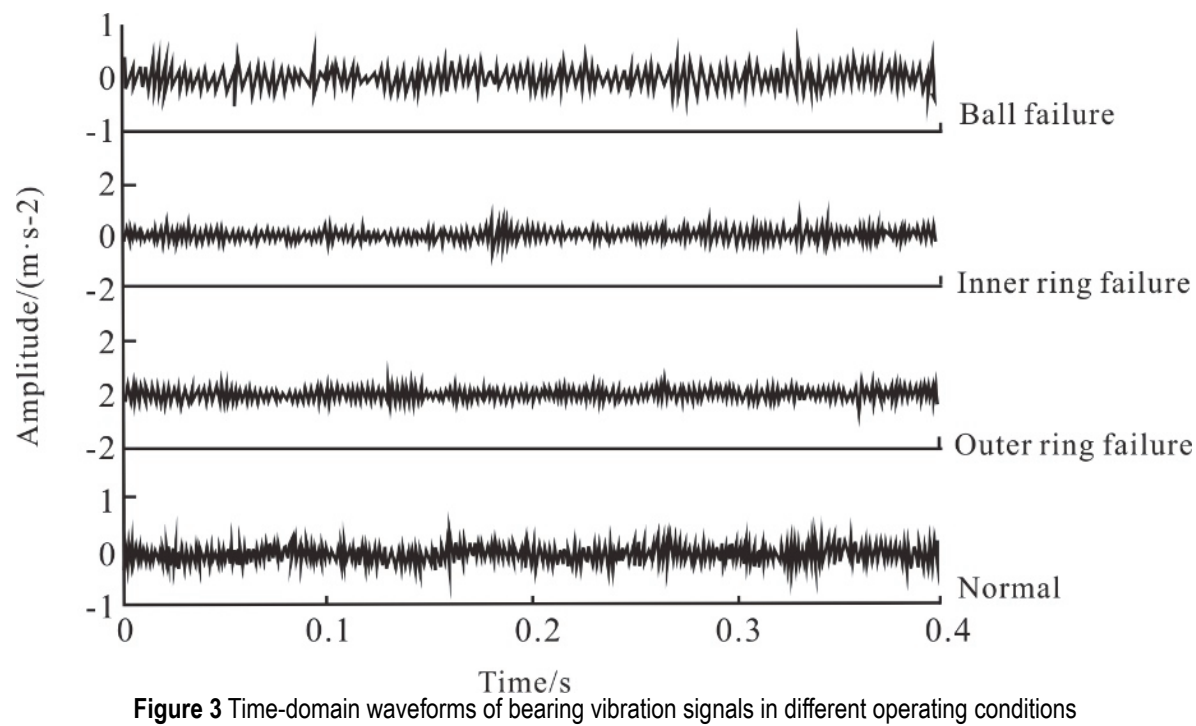

For the above samples, the following experiment steps are performed:

1) Select 20 of each state randomly as the training samples, and the remaining 30 as the experiment samples.

Calculate the CMPE of each sample, take the embedding dimension $m=4$, scale factor $\tau=25$, and delay $\lambda=1$ to establish the original high-dimensional feature set $R^{200 \times 25}$.

Table 1 Identification results of the classifier

\begin{tabular}{|c|c|c|c|c|c|c|c|c|c|c|}
\hline \multirow{2}{*}{\multicolumn{2}{|c|}{ Method }} & \multicolumn{3}{|c|}{ PCA } & \multicolumn{3}{|c|}{ LTSA } & \multicolumn{3}{|c|}{ FLVPLA } \\
\hline & & GA-SVM & PSO-SVM & BA-SVM & GA-SVM & PSO-SVM & BA-SVM & GA-SVM & PSO-SVM & BA- \\
\hline \multirow{6}{*}{$\begin{array}{c}\text { Fault } \\
\text { identificati } \\
\text { on rate } / \%\end{array}$} & Fault of the steel ball & 76.67 & 80 & 120 & 93.33 & 100 & 100 & 96.67 & 96.67 & 100 \\
\hline & Fault of the inner ring & 76.67 & 63.33 & 60 & 100 & 86.37 & 96.17 & 100 & 100 & 100 \\
\hline & Fault of the outer ring & 100 & 100 & 110 & 100 & 100 & 100 & 100 & 100 & 100 \\
\hline & Normal & 100 & 100 & 120 & 100 & 100 & 100 & 100 & 100 & 100 \\
\hline & Average identification rate & 88.33 & 85.83 & 91.5 & 98.33 & 96.77 & 99.07 & 99.17 & 9917 & 100 \\
\hline & Running time/s & 16.57 & 295 & 2.49 & 15.02 & 2.65 & 2.30 & 15.29 & 2.68 & 2.62 \\
\hline
\end{tabular}

2) The labeled information and non-labeled information in the training samples are randomly allocated at a ratio of 2:3. Input the original high-dimensional fault feature set into FLVPLA for dimensionality reduction, and conduct comparative analysis based on the principal component analysis (PCA) and LTSA. For data visualization, let the target dimension $d=3$, determine the optimal nearest neighbor parameter of FLVPLA $k_{1}=95$, and the optimal nearest neighbor parameter of LTSA $k_{2}=$ 76 through multiple experiments. From the figure, it can be observed that PCA has a poor clustering effect on outer ring faults. Although effective clustering of steel ball faults, inner ring faults and normal conditions is accomplished, serious mode mixing occurs in both the inner ring faults and the steel ball faults. The LTSA method can be used to separate the 4 types of samples roughly. However, there is still a certain amount of mode mixing between the inner ring faults and the steel ball faults, whereas FLVPLA can completely separate the 4 types of faults, with a higher classification degree. Compared with PCA and LTSA, the 
aggregation in each state is improved, and the dimensionality reduction effect is relatively ideal.

3) In order to further verify the dimensionality reduction effect of FLVPLA and the feasibility and effectiveness of BA-SVM, the three low-dimensional feature sets obtained are input into different classifiers for pattern identification. The results are shown in Tab. 1 as the following. Among them, the bat population size of BASVM is set to 10 , the impulse loudness is set to 0.5 , the pulse rate is set to 0.5 , and the maximum number of iterations is set to 100 . The population size of GA-SVM is set to 20 , the crossover probability is set to 0.9 , the mutation probability is set to 0.05 , and the number of evolutionary iterations is set to 200 . The population size of PSO-SVM is set to 10 , the learning factor is set to $c_{1}=c_{2}$ $=2$, and the number of iterations is set to 200 .

From Tab. 1, it can be seen that since PCA is a linear dimensionality reduction method, it aims to transform high-dimensional data into the direction of the maximum variance set of the data. However, the nonlinear structure of the original feature set is neglected. Hence, the average identification rate of PCA after dimensionality reduction is lower than that of LTSA. LTSA is an unsupervised learning method, which does not take the category information of the original sample into consideration. As a result, its identification rate is to be lower than that of FLVPLA due to part of the mode mixing phenomena. In the FLVPLA with improved distance equation, a metric function that combines cosine similarity and Euclidean distance is used to calculate the distance between sample points. The position and angle information between the sample points are fused, the distance obtained is more accurate, and the obtained low-dimensional features can keep a higher original feature manifold structure. Hence, the average identification rate is higher, and the identification rate of the input BA-SVM classifier can achieve $100 \%$. No matter which dimensionality reduction method is used, when different classifiers, the identification rate of BA-SVM is higher than that of the GA-SVM and PSO-SVM, and the calculation time is significantly shorter, which suggests that the bat algorithm optimization support vector machine in the bearing fault has the advantages in the diagnosis applications.

\section{DISCUSSION}

In order to further verify the effectiveness of the method proposed in this paper, the bearing data is used for analysis. The motor speed is $1730 \mathrm{r} / \mathrm{min}$, the load condition is $1 \mathrm{HP}$, the experiment bearing is a $6205-2 \mathrm{RS}$ deep groove ball bearing, and the EDM technology is used to simulate the fault of the bearing. The fault diameters are $0.178 \mathrm{~mm}$ (mild fault) and $0.356 \mathrm{~mm}$ (moderate fault), respectively. There are a total of 7 types of signals with 2 different levels of the fault and normal state, that is, mild fault of the steel ball (BOF1), mild fault of the inner ring (IRF1), mild fault of the outer ring (ORF1), moderate fault of the steel ball (BOF2), moderate fault of the inner ring (IRF2), moderate fault of the outer ring (ORF2), and normal status (NOR). Fifty samples are extracted from each state, and each sample is 2048 in length. Twenty samples are randomly selected from each state as training samples, and the remaining 30 are used as experiment samples. The CMPE value of each sample is calculated, the embedding dimension $m=4$, scale factor $\tau=25$, and delay $\lambda=1$ are selected to establish the original high-dimensional feature set $R^{350 \times 25}$.

The labeled information and non-labeled information in the training samples are randomly assigned at a ratio of $2: 3$. The original high-dimensional fault feature set is input into FLVPLA for dimensionality reduction, and the principal component analysis (PCA) and LTSA are used for comparative analysis. For the visualization of data, the target dimension $d=3$ is set. The optimal neighbor parameter $k_{1}=54$ of FLVPLA and the optimal neighbor parameter $k_{2}=52$ of LTSA are determined through multiple experiments.

From the results, it can be known that in the lowdimensional subset after dimensionality reduction based on PCA, three types of samples with the mild fault of the steel ball, mild fault of the outer ring, and moderate fault of the steel ball have shown more serious mode mixing phenomena. Although the LTSA method can be used to separate the 7 types of samples roughly, there is still a certain volume of mode mixing phenomena in the samples with moderate faults in the inner ring and the samples with moderate faults in the outer ring; whereas FLVPLA can completely separate the 7 types of faults and presents a high degree of classification, with a relatively ideal dimensionality reduction effect.

In order to verify the dimensionality reduction effect of FLVPLA, the set of low-dimensional features obtained based on the three dimensionality reduction methods are input into the classifier for fault identification. According to statistics, PCA has incorrectly determined 6 sample categories, with an average identification rate of $97.14 \%$ $(204 / 210)$. LTSA has incorrectly determined 3 sample categories, with an average identification rate of $98.57 \%$ (207/210). The predicted results of FLVPLA are entirely consistent with the actual results, with a fault identification rate of $100 \%$. The analysis results have further verified the superiority of the rolling bearing fault diagnosis method based on the CMPE, FLVPLA, and BA-SVM.

\section{CONCLUSION}

In order to improve the accuracy of bearing fault diagnosis, a bearing fault diagnosis method based on fuzzy logic variable phase hierarchical algorithm (flvpla) is established in this paper. By calculating the eigenvalues of vibration signals, the original gauvitt collection is constructed. The low viterbilt collection after dimensionality reduction is classified. Experiments show that the recognition accuracy of this method is higher than GA-SVM and PSO-SVM. It can effectively warn the bearing fault, so as to improve the utilization rate of wind turbine and reduce the loss as much as possible. It has great practical engineering application value.

\section{REFERENCES}

[1] Alamdari, M. M., Kildashti, K., Samali, B., \& Goudarzia, H. V. (2019). Damage diagnosis in bridge structures using rotation influence line: Validation on a cable-stayed bridge. Engineering Structures, 185(15), 1-14. https://doi.org/10.1016/j.engstruct.2019.01.124 
[2] Bekkers, A., Borren, N., Ederveen, Fokkinga, E., Andrade De Jesus, D., Sánchez Brea, Luisa., Stefan, K., van Walsum, T., Barbosa-Breda, J., \& Stalmans, I. (2020). Microvascular damage assessed by optical coherence tomography angiography for glaucoma diagnosis: a systematic review of the most discriminative regions. Acta Ophthalmologica, 98(6), 1-8. https://doi.org/10.1111/aos.14392

[3] Malega, P., Kadarova, J., \& Kobulnicky, J. (2017). Improvement of Production Efficiency of Tapered Roller Bearing by Using Plant Simulation. International Journal of Simulation Modelling, 16(4), 682-693. https://doi.org/10.2507/IJSIMM16(4)10.405

[4] Chegini, S. N., Manjili, M. J. H., \& Bagheri, A. (2020). New fault diagnosis approaches for detecting the bearing slight degradation. Meccanica, 55(1), 261-286. https://doi.org/10.1007/s11012-019-01116-x

[5] Kiran, M., Jinah, K., Aleodor, A. A., Tricia, A. M., Roberto, A. N., Claudia, I. V., Maxwell, A. F., \& Patrick. O. E. (2020) TERT and TERT promoter in melanocytic neoplasms: Current concepts in pathogenesis, diagnosis, and prognosis. Journal of Cutaneous Pathology, 47 (6122), 108-115. https://doi.org/10.1111/cup.13691

[6] Agahi, H. \& Mahmoodzadeh, A. (2020). Decision fusion scheme for bearing defects diagnosis in induction motors. Electrical Engineering, 5 (4), 54-60. https://doi.org/10.1007/s00202-020-01024-4

[7] Wang, H., Chen, J., Zhou, Y., \& Guangxian, N. (2020). Early fault diagnosis of rolling bearing based on noise-assisted signal feature enhancement and stochastic resonance for intelligent manufacturing. The International Journal of Advanced Manufacturing Technology, 1 (4), 1-7. https://doi.org/10.1007/s00170-019-04333-6

[8] Ambrozkiewicz, B., Litak, G., Georgiadis, A., Syta, A., Meier, N., \& Gassner, A. (2021). Effect of Radial Clearance on Ball Bearing's Dynamics Using a 2-DOF Model. International Journal of Simulation Modelling, 20(3), 513524. https://doi.org/10.2507/IJSIMM20-3-568

[9] Lv, Y., Ge, M., Zhang, Y., Cancan, Y., \& Yubo, M. (2019). A Novel Demodulation Analysis Technique for Bearing Fault Diagnosis via Energy Separation and Local Low-Rank Matrix Approximation. Sensors, 19 (17), 3755-3760. https://doi.org/10.3390/s19173755

[10] Ma, S. \& Chu, F. (2019). Ensemble deep learning-based fault diagnosis of rotor bearing systems. Computers in Industry, 105(3), 143-152. https://doi.org/10.1016/j.compind.2018.12.012

[11] Liu, Y. Z., Zou, Y. S., Jiang, Y. L., Hui, Y., \& Guo-Fu, D. (2020). A Novel Method for Diagnosis of Bearing Fault Using Layered Multitasks Convolutional Neural Networks. Shock and Vibration, 20(13), 1-14. https://doi.org/10.1155/2020/8846822

[12] Li, X., Zhang, W., \& Ding, Q. (2019). Understanding and improving deep learning-based rolling bearing fault diagnosis with attention mechanism. Signal Processing, 161(8), 136-154. https://doi.org/10.1016/j.sigpro.2019.03.019

[13] Yong, L., Rui, Y., Tao, W., Hewenxuan, L., \& Gangbing, S. (2018). Health Degradation Monitoring and Early Fault Diagnosis of a Rolling Bearing Based on CEEMDAN and Improved MMSE. Materials, 11(6), 1009-1014. https://doi.org/10.3390/ma11061009

[14] Shao, H., Lin, J., Zhang, L., \& Muheng, W. (2020). Compound fault diagnosis for a rolling bearing using adaptive DTCWPT with higher order spectra. Quality Engineering, 7(2), 1-12. https://doi.org/10.1080/08982112.2020.1749654

[15] Ming, Zhang, Duo, Weining, L., Jun, Y., Zhiheng, L., \& Bin, L. (2019). A Deep Transfer Model with Wasserstein Distance Guided Multi-Adversarial Networks for Bearing Fault Diagnosis Under Different Working Conditions. IEEE Access, 7(5), 65303-65318.
https://doi.org/10.1109/ACCESS.2019.2916935

[16] Fei, S., Chao, C., Jiawen, X., et al. (2018). A New Spectral Centroid Transfer Strategy for Bearing Fault Diagnosis under Varying Working Conditions. Yi Qi Yi Biao Xue Bao/Chinese Journal of Scientific Instrument, 28(4), 1-8.

[17] Zhang, M., Wang, D., Lu, W., et al. (2019). A Deep Transfer Model with Wasserstein Distance Guided Multi-Adversarial Networks for Bearing Fault Diagnosis Under Different Working Conditions. IEEE Access, 8(4), 101-110.

Contact information:

\section{Yao CHEN}

ShangLuo University,

Shannxi, ShangLuo, 726000, China

Email: youyouzo@163.com 